\title{
Systemic antimicrobials offer an additional benefit over scaling and root planing alone in the treatment of periodontitis
}

\author{
Do adjunctive systemic antimicrobials improve the clinical outcome of scaling \\ and root planing in the treatment of periodontitis?
}

\begin{abstract}
Herrera D, Sanz M, Jepsen S, Needleman I, Rolda'n S.
A systematic review on the effect of systemic antimicrobials as an adjunct to scaling and root planing in periodontitis patients. J Clin Periodontol 2002; 29(Suppl. 3):S136-S159
\end{abstract}

Data sources MEDLINE, the Cochrane Oral Health Group Trials Register and EMbase were data sources. Reference lists from relevant articles were also hand-searched and a further search was made by hand of selected journals dated up to April 2001.

Study selection Randomised controlled clinical trials or controlled clinical trials of at least 6 months' duration were selected in which systemically healthy patients with either aggressive periodontitis (AgP) or chronic periodontitis (CP) were treated by scaling and root planing (SRP) plus systemic antimicrobials, or by SRP alone or with placebo, for a minimum of 6 months. Clinical attachment level (CAL) change and probing pocket depth (PPD) change were the main outcome measures. Data extraction and synthesis Information regarding quality and study characteristics was extracted independently by two reviewers. Kappa scores determined their agreement. Results were collated by drug, disease and PPD category. Data was pooled (when mean differences and standard errors were available), using either a fixedeffects or random-effects model for meta-analysis.

Results A total of 25 papers were included in the analysis. Overall, SRP plus systemic antimicrobial groups demonstrated better results in CAL and PPD change than SRP alone and than placebo groups. Randomisation and allocation-concealment methods were seldom reported and blindness was usually not defined clearly. The studies showed high variability and insufficient relevant information for an adequate assessment. As a result only limited meta-analyses could be performed. These showed a statistically significant additional benefit of spiramycin (PPD change) and amoxicillin/metronidazole (CAL change) in deep pockets.

Conclusions Systemic antimicrobials in conjunction with SRP can offer an additional benefit over SRP alone in the treatment of periodontitis, in terms of CAL and PPD change and reduced risk of additional CAL loss. Differences in study methodology and lack of data, however, precluded an adequate and complete pooling of data for more comprehensive analyses. It was difficult to establish definitive conclusions, although people who had greater pocket-depth, progressive or active disease, or a specific microbiological profile, may benefit more from this adjunctive therapy.

\section{Commentary}

The educational, clinical, political and financial implications of this landmark study are significant. The results imply that periodontal disease may be treated more effectively by scaling plus antibiotics than by scaling alone. If true, this would demand educational changes in both pre- and postdoctoral curricula, and could also create political turmoil between, for example, the practising general dentists who currently provide $80 \%$ of the periodontal care in the US and the periodontists and oral surgeons who provide $20 \%$ of the care. These findings could also create financial changes as insurers begin to reconsider the benefits they will provide for periodontal care.

The key statement in the previous paragraph is, "if true". Although statistically significant improvements were noted following antibiotic use, the differences were not clinically significant. Further, the selection of specific antibiotics was not delineated. Thus, despite these findings suggesting large changes are needed in periodontal care, these changes await the systematic reviews that would confirm them and, secondly, additional specificity in antibiotic selection. Also of import are antecedent systematic reviews demonstrating the clinical benefit of scaling alone. ${ }^{1,2}$

\section{Practice point}

- The review indicates that periodontal disease may be more effectively treated with scaling and antibiotics than by scaling alone.

\section{Richard Niederman}

DSM-Forsyth Center for Evidence-Based Dentistry, The Forsyth Institute, and the Department of Health Policy and Health Services Research, Boston University Goldman School of Dental Medicine, Boston, Massachusetts, USA

1. Elley J, Gold L, Burls A, Gray M. Scale and Polish for Chronic Periodontal Disease. Birmingham: West Midlands Health Technology Assessment Group, Department of Public Health and Epidemiology, University of Birmingham; 2001.

2. Hung HC, Douglass CW. Meta-analysis of the effect of scaling and root planing, surgical treatment and antibiotic therapies on periodontal probing depth of attachment loss. J Clin Periodontol 2002; 29:975-986.

Evidence-Based Dentistry (2003) 4, 62.

doi:10.1038/sj.ebd.6400197 